\title{
Research Article \\ Electron Spin Resonance Measurement with Microinductor on Chip
}

\begin{abstract}
Akio Kitagawa
Division of Electrical Engineering and Computer Science, Kanazawa University, Kakuma-machi, Kanazawa 920-1192, Japan

Correspondence should be addressed to Akio Kitagawa, kitagawa@is.t.kanazawa-u.ac.jp

Received 6 May 2011; Revised 7 July 2011; Accepted 11 July 2011

Academic Editor: Csaba Dücső

Copyright (C) 2011 Akio Kitagawa. This is an open access article distributed under the Creative Commons Attribution License, which permits unrestricted use, distribution, and reproduction in any medium, provided the original work is properly cited.

The detection of radicals on a chip is demonstrated. The proposed method is based on electron spin resonance (ESR) spectroscopy and the measurement of high-frequency impedance of the microinductor fabricated on the chip. The measurement was by using a frequency sweep of approximately $100 \mathrm{MHz}$. The ESR spectra of di(phenyl)-(2,4,6-trinitrophenyl)iminoazanium (DPPH) dropped on the microinductor which is fabricated with CMOS 350-nm technology were observed at room temperature. The volume of the $\mathrm{DPPH}$ ethanol solution was $2 \mu \mathrm{L}$, and the number of spins on the micro-inductor was estimated at about $10^{14}$. The sensitivity is not higher than that of the standard ESR spectrometers. However, the result indicates the feasibility of a near field radical sensor in which the microinductor as a probe head and ESR signal processing circuit are integrated.
\end{abstract}

\section{Introduction}

Electron spin resonance (ESR) spectroscopy or electron paramagnetic resonance (EPR) spectroscopy is a widely used way of measuring and characterizing electronic materials $[1,2]$. It is also used in biomedical analysis and imaging $[3,4]$ because radicals can be identified and quantitatively determined. The ESR phenomenon occurs based on the Zeeman effect of the unpaired electron in radicals, which can be observed by the resonance absorption of the magnetic wave at the specified frequency. The ESR frequency of the magnetic wave is proportional to the applied DC magnetic field. The ESR spectrum is provided by a frequency sweep of the magnetic wave or a sweep of the DC magnetic field strength. However, sweeping the DC magnetic field is widely used in most cases because the resonator which is designed to make resonance at the specific frequency is used to detect the small absorption of the electromagnetic power in the resonance [5]. In ESR spectroscopy on the frequency axis, the control system of the DC magnetic field can be eliminated, and this enables development of the radical sensors which integrate the ESR measurement circuit.

In this paper, we demonstrate ESR measurement with a microinductor to show the possible integration of ESR signal processing on a chip. The ESR spectroscopy was done by a frequency sweep of around $100 \mathrm{MHz}$ in the fixed magnetic field.

\section{Principle of Measurement}

The configuration of the measurement system is shown in Figure 1. The high-frequency current in the microinductor generates a high-frequency magnetic wave. The DC magnetic field is perpendicularly applied to the microinductor. The magnetic permeability of the sample put on the microinductor is affected by the ESR, and the variation of the magnetic permeability is then detected as a variation of the impedance of the microinductor. The high-frequency magnetic field generated by the microinductor includes the vertical and the horizontal component against the DC magnetic field on the surface of the microinductor. The magnetic permeability is strongly affected by the perpendicular component of the high-frequency magnetic field [6], which is concentrated in the proximity of the metal wire to constitute the microinductor. The spatial range of the measurement depends on the intensity of the high-frequency current and the width of the metal wire. 


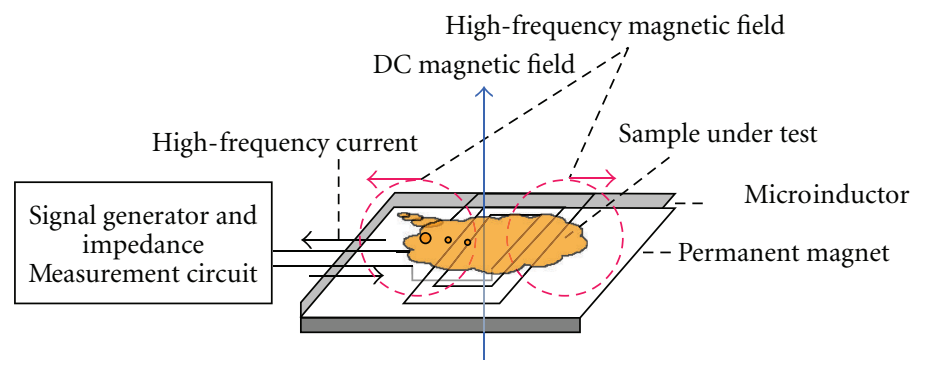

FIgURE 1: Structure of measurement system.

The relative permeability $\mu(\omega)$ is expressed as a function of the frequency as shown by:

$$
\mu(\omega)=\mu_{r}(\omega)-j \mu_{i}(\omega),
$$

where $j$ is an imaginary unit, $\mu_{r}$ and $\mu_{i}$ are a real and an imaginary part of the magnetic permeability. The impedance of the microinductor affected by the paramagnetic materials is expressed as follows (2):

$$
Z(\omega)=R_{0}+j \omega\{1+\alpha \mu(\omega)\} L_{0},
$$

where $R_{0}$ and $L_{0}$ are a resistance and an inductance of the microinductor, which is not affected by the sample material. Here, $\alpha$ is a filling factor which is a parameter of the contribution of the sample material to the magnetic permeability. From (1) and (2), (3a) is derived,

$$
\begin{gathered}
Z(\omega)=\left(R_{0}+\omega \alpha \mu_{i} L_{0}\right)+j \omega\left(L_{0}+\alpha \mu_{r} L_{0}\right), \\
\stackrel{\text { def }}{=}\left(R_{0}+R_{\mathrm{ESR}}\right)+j \omega\left(L_{0}+L_{\mathrm{ESR}}\right) .
\end{gathered}
$$

Here, $R_{\mathrm{ESR}}$ and $L_{\mathrm{ESR}}$ are a resistance component and an inductance component which are derived from the permeability of the sample material and proportional to the inductance of the microinductor. The impedance $Z(\omega)$ can be calculated from the measurement of the $S$-parameters of the microinductor by using

$$
Z(\omega)=Z_{0} \frac{1+S_{11}-S_{12} S_{21} /\left(1+S_{22}\right)}{1-S_{11}+S_{12} S_{21} /\left(1+S_{22}\right)} .
$$

Here, $Z_{0}$ is the characteristic impedance of the ports of the measurement instruments. The characteristic impedance of the commercially available measurement instruments and connectors is normally $50 \mathrm{ohm}$.

\section{Measurement Results}

The microinductor of a 2-port configuration was fabricated with CMOS $350 \mathrm{~nm}$ technology. This microinductor was designed to have a higher self-resonance frequency than a measurement band. The wire metal was aluminum with a thickness of $570 \mathrm{~nm}$ and covered by the $\mathrm{Si}_{3} \mathrm{~N}_{4}$ passivation layer. A photograph and specification of the microinductor are shown in Figure 2 and Table 1, respectively. The measured resistance $\left(R_{0}\right)$ and inductance $\left(L_{0}\right)$ are $16.2 \mathrm{ohm}$ and $15.8 \mathrm{nH}$ at a frequency of $100 \mathrm{MHz}$, respectively.

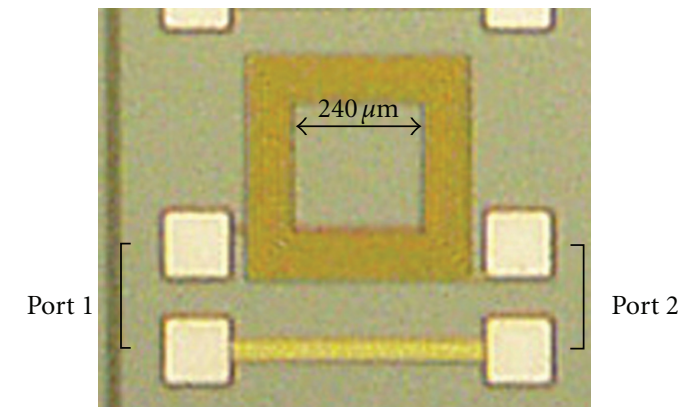

FIgUre 2: Photograph of microinductor.

TABle 1: Specification of microinductor.

\begin{tabular}{lcccc}
\hline Shape & $\begin{array}{c}\text { Inner diameter Number Line width } \\
{[\mu \mathrm{m}]}\end{array}$ & $\begin{array}{c}\text { Line } \\
\text { of turns } \\
{[\mu \mathrm{m}]}\end{array}$ & $\begin{array}{c}\text { spacing } \\
{[\mu \mathrm{m}]}\end{array}$ \\
\hline Rectangular spiral & 240 & 6 & 10 & 1 \\
\hline
\end{tabular}

The measurement results for di(phenyl)-(2,4,6-trinitrophenyl)iminoazanium (DPPH) are shown in Figure 3. DPPH is the popular standard of the position ( $g$-marker) and intensity of EPR signals. The $R_{\mathrm{ESR}}$ and $L_{\mathrm{ESR}}$ were calculated from the measured $S$-parameters by using (3b) and (4). The 2$\mu \mathrm{L}$ DPPH ethanol solution $(0.1 \mathrm{mg} / \mathrm{L})$ was dropped on a microinductor and dried at room temperature. The number of spins on the microinductor was estimated at about $10^{14}$. The magnetic field was $4.2 \mathrm{mT}$ and the center frequency of the signal was $117.6638 \mathrm{MHz}$. From these results, the $g$-factor of the ESR line was estimated at 2.0016. The estimated $g$ factor is smaller than the reported value $(g=2.0023)$ [7]. As the magnetic field is not measured accurately enough to estimate the $g$-factor, calibration by another $g$-marker is needed to estimate the $g$-factor more precisely.

\section{Conclusions}

The ESR measurement with a microinductor fabricated on a chip is demonstrated to show the possible integration of ESR signal processing on a chip. The ESR spectra of DPPH are observed at room temperature. The number of spins on the microinductor was estimated at about $10^{14}$. While sensitivity is not higher than that of the standard ESR spectrometers, the measurement system with a microinductor has several 


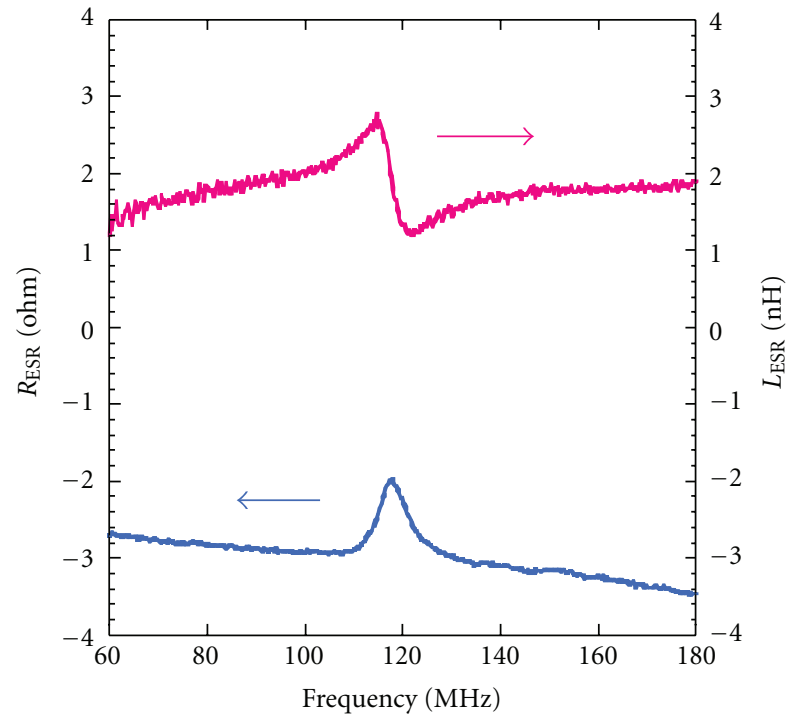

FIGURE 3: Spectra of $R_{\mathrm{ESR}}$ and $L_{\mathrm{ESR}}$ for DPPH at room temperature. Magnetic field was $4.2 \mathrm{mT}$.

advantages. First, it is of small size, simple, and compact. Second, frequency scans can be done without the need for a magnetic field sweep. Third, the instrumentation is well developed in the very high frequency range (from $30 \mathrm{MHz}$ to $300 \mathrm{MHz}$ ) and the ultrahigh frequency range (from $300 \mathrm{MHz}$ to $3 \mathrm{GHz}$ ). Fourth, the probe head can be manufactured with CMOS technology. This instrument may be used to construct near-field ESR apparatuses with high spatial resolution.

\section{Acknowledgments}

This work was supported by VLSI Design and Education Center (VDEC), The University of Tokyo in collaboration with Cadence Corporation and Mentor Graphics, Inc. The VLSI chip in this study has been fabricated in the chip fabrication program of VDEC, the University of Tokyo in collaboration with Rohm Corporation and Toppan Printing Corporation. This work was also supported by Grant-in-Aid for Scientific Research (C) (20510116) of Japan Society for the Promotion of Science (JSPS) and Adaptable \& Seamless Technology Transfer Program through Target-driven R\&D (AS2121327A) of Japan Science and Technology Agency (JST).

\section{References}

[1] N. A. Schultz and P. C. Taylor, "Temperature dependence of the optically induced production and annealing of silicon dangling bonds in hydrogenated amorphous silicon," Physical Review B-Condensed Matter and Materials Physics, vol. 65, no. 23, Article ID 235207, pp. 2352071-2352078, 2002.

[2] M. Oubaha, R. Copperwhite, C. McDonagh, P. Etienne, and B. D. MacCraith, "Structural and optical characterisation of an Erbium/Ytterbium doped hybrid material developed via a nonhydrolytic sol-gel route," SRX Materials Science, vol. 2010, Article ID 101747, 2010.
[3] S. A. Dikanov, B. D. Liboiron, K. H. Thompson et al., "One and two dimensional pulsed electron paramagnetic resonance studies of in vivo vanadyl coordination in rat kidney," Bioinorganic Chemistry and Applications, vol. 1, no. 1, pp. 69-83, 2003.

[4] C. D. Dharmaraj, K. Thadikonda, A. R. Fletcher et al., "Reconstruction for time-domain in vivo EPR 3D multigradientoximetricimaging-a parallel processing perspective," International Journal of Biomedical Imaging, vol. 2009, Article ID 528639, 2009.

[5] J. J. Henderson, C. M. Ramsey, H. M. Quddusi, and E. Del Barco, "High-frequency microstrip cross resonators for circular polarization electron paramagnetic resonance spectroscopy," Review of Scientific Instruments, vol. 79, no. 7, Article ID 074704, pp. 1-4, 2008.

[6] J. S. Toll, "Causality and the dispersion relation: logical foundations," Physical Review, vol. 104, no. 6, pp. 1760-1770, 1956.

[7] B. C. Gilbert, M. J. Davies, and K. A. McLauchlan, "Electron paramagnetic resonance," The Royal Society of Chemistry, vol. 17 , p. $178,2000$. 

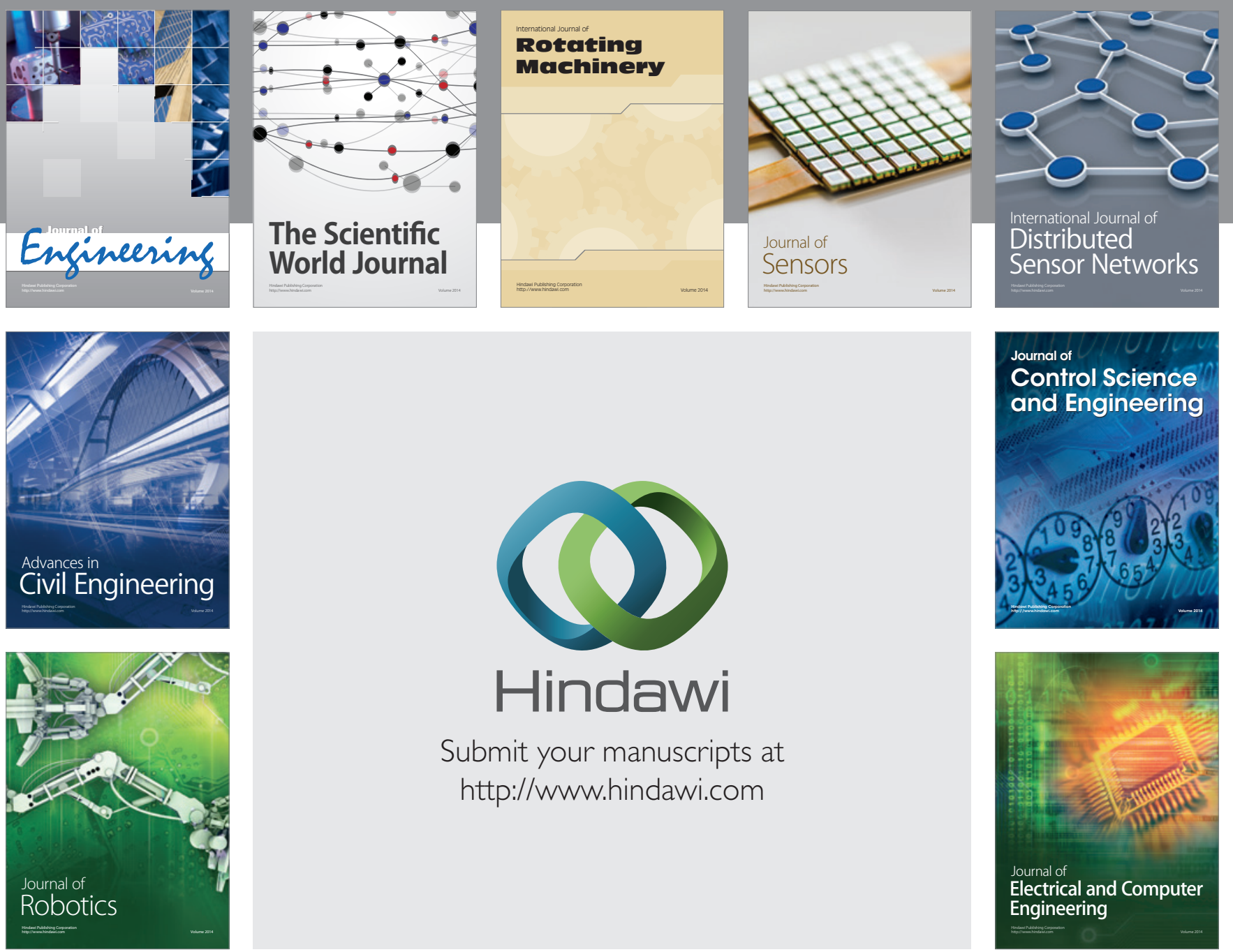

Submit your manuscripts at

http://www.hindawi.com
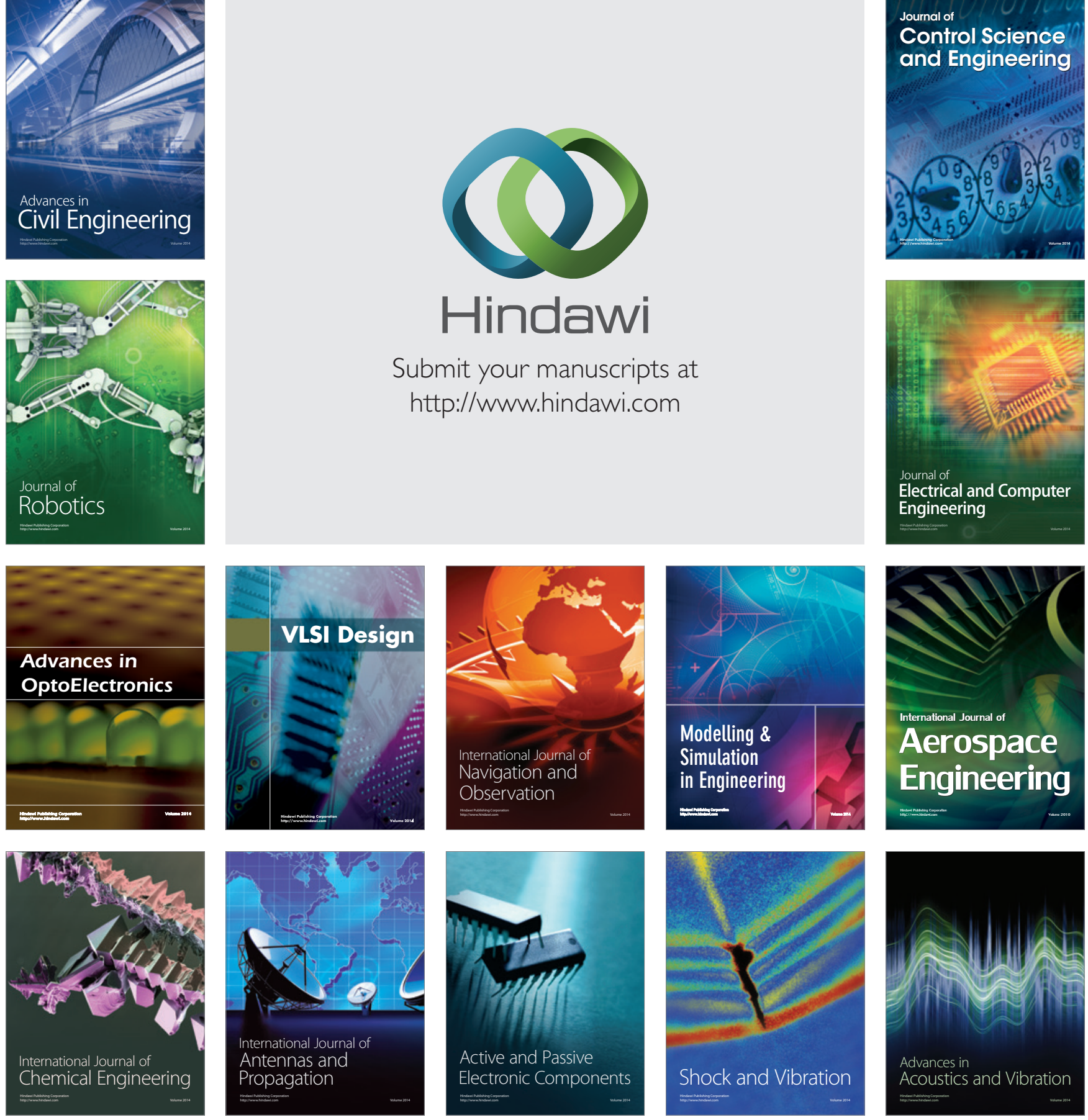